\title{
Crystal structure of 1-cyclopropyl-6-fluoro-8-methoxy-7-(3-methyl- 1-piperazinyl)-1,4-dihydro-4-oxo-3-quinolinecarboxylic acid hydrochloride, $\left[\mathrm{C}_{19} \mathrm{H}_{23} \mathrm{FN}_{3} \mathrm{O}_{4}\right] \mathrm{Cl}$
}

\author{
Liang-Cai $\mathrm{Yu}^{*}$, Yong Xia and Sheng-Li Liu \\ Engineering of Hunan University of Science and Technology, College of Chemistry \& Chemical, Xiangtan, P. R. China
}

Received October 16, 2008, accepted and available on-line March 12, 2009; CCDC no. 1267/2481
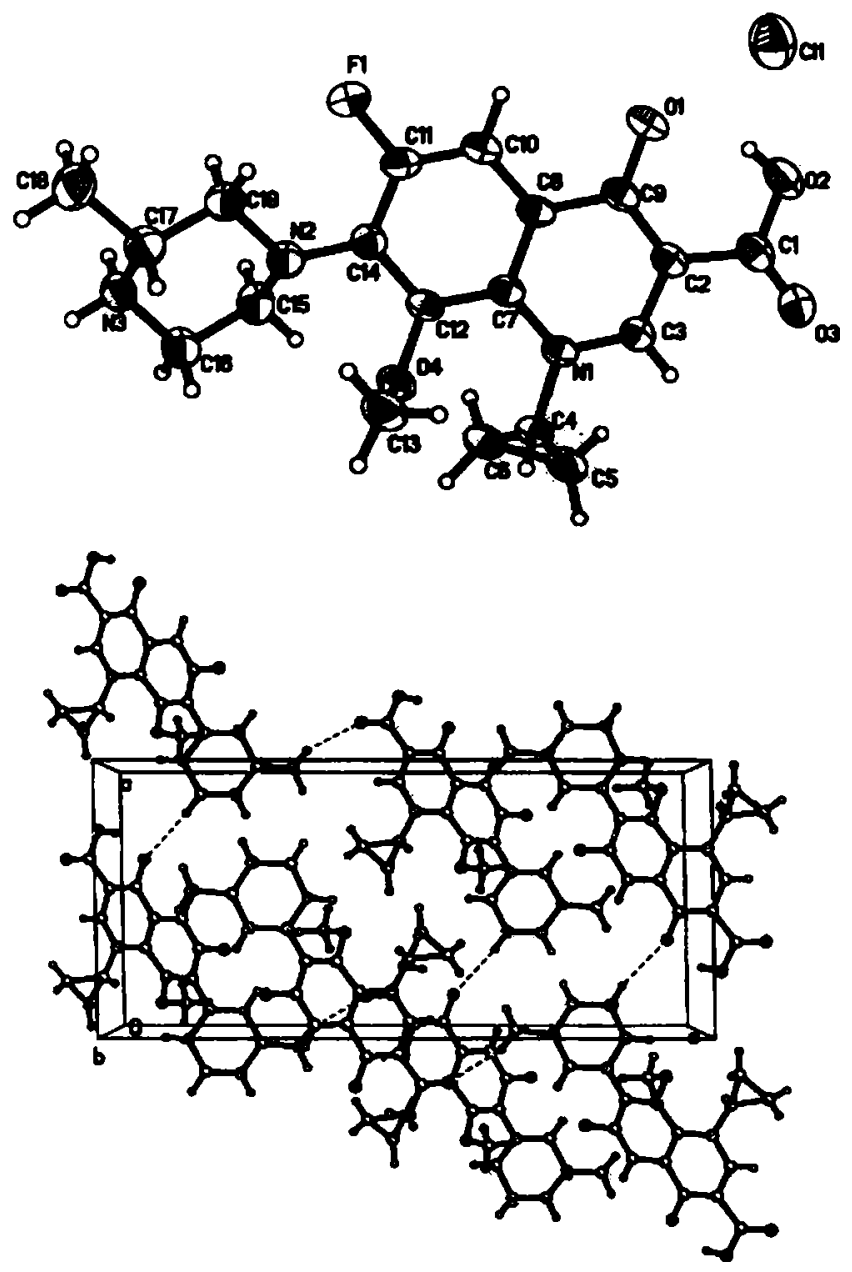

Abstract

$\mathrm{C}_{19} \mathrm{H}_{23} \mathrm{ClFN}_{3} \mathrm{O}_{4}$, monoclinic, $P 121 / n 1$ (no. 14), $a=10.833(4) \AA, b=7.683(2) \AA, c=23.976(8) \AA$, $\beta=91.11(2)^{\circ}, V=1995.1 \AA^{3}, Z=4, R_{g r}(F)=0.050$, $w R_{\text {ref }}\left(F^{2}\right)=0.157, T=296 \mathrm{~K}$.

\section{Source of material}

Gatifloxacin (HGat, $0.8 \mathrm{mmol}, 0.3004 \mathrm{~g})$ and $\mathrm{FeCl}_{3}(0.5 \mathrm{mmol}$, $0.0650 \mathrm{~g}$ ) were thoroughly mixed and placed into autoclave. After addition of $5 \mathrm{~mL}$ of $\mathrm{EtOH}$ and $15 \mathrm{~mL}$ of $\mathrm{H}_{2} \mathrm{O}$, the $\mathrm{pH}$ value of the solution was adjusted to 6.0 . The autoclave was heated to $120^{\circ} \mathrm{C}$ for 3 days to obtain pale yellow crystals of the title compound.

* Correspondence author (e-mail: ylcphd@gmail.com)

\section{Discussion}

In the title crystal structure, the bicyclical quinoline ring system is essentially planar, whereas the piperazine ring has a chair conformation (figure, top). The oxygen atom 02 of cartoxylate group of quinolones is protonated, The terminal nitrogen atom N3 of the piperazine group is also protonated. The positive charge of $\mathbf{H}_{2}$ Gat is neutralized by one chloride anion. The cyclopropyl group plane (C4, C5 and C6) is not orthogonalto the mean plane of the quinolone system; the corresponding dihedral angle is $61.9^{\circ}$. The $-\mathrm{OCH}_{3}$ group is sticking out of the plane with torsion angles of 100.3(3) for C13-04-C12-C7. The oxygen atom $\mathrm{O} 2$ of carboxylate group of quinolones is protonated, and this proton is bonded to the oxygen $\mathrm{Ol}$ of the carbonyl group viaintramolecular hydrogen $02-\mathrm{DH} 2 \ldots \mathrm{O} 1$, forming a six membered pseudo-ring $(\mathrm{O} 1 / \mathrm{C} 9 / \mathrm{C} 2 / \mathrm{C} 1 / \mathrm{O} 2 / \mathrm{H} 2)$. The intermolecular hydrogen bond N3$\mathrm{H} 3 \cdots \mathrm{Cll}^{\mathrm{i}}$ (symmetry code $\mathrm{i}:-x+1 / 2, y+1 / 2,-z+1 / 2$ ) and N3H3B $\cdots \mathrm{Cl}^{\text {ii }}$ (symmetry code ii: $x-1, y+1, z$ ) assembly the residues to form a 3D network (figure, bottom).

Table 1. Data collection and handling.
Crystal:

Wavelength:

$\mu$ :

Diffractometer, scan mode:

$2 \theta_{\max }:$

$N(h k l)_{\text {measured, }} N(h k l)_{\text {unique: }}$ Criterion for $I_{\text {obs }}, N(h k l)_{\text {gt }}$ :

$N($ param) refined:

Programs: colorless block, size $0.14 \times 0.18 \times 0.50 \mathrm{~mm}$ Mo $K_{\alpha}$ radiation ( $\left.0.71073 \hat{\AA}\right)$ $2.31 \mathrm{~cm}^{-1}$

Bruker SMART CCD, $\varphi / \omega$

$52.02^{\circ}$

11883,3887

$I_{\text {obs }}>2 \sigma\left(I_{\text {obs }}\right), 2416$

257

SHELXS-97 [1], SHELXL-97 [2], SHELXTL [3]
Table 2. Atomic coordinates and displacement parameters (in $\AA^{2}$ ).

\begin{tabular}{llrlrl}
\hline Atom & Site & \multicolumn{1}{l}{$x$} & \multicolumn{1}{l}{$y$} & \multicolumn{1}{l}{$U_{\text {iso }}$} \\
\hline H(3A) & $4 e$ & -0.1962 & 0.9796 & 0.2466 & 0.078 \\
H(3B) & $4 e$ & -0.1231 & 1.1318 & 0.2325 & 0.078 \\
H(2) & $4 e$ & 0.7403 & 0.8490 & 0.0286 & 0.121 \\
H(3) & $4 e$ & 0.4279 & 0.6398 & -0.0382 & 0.063 \\
H(4) & $4 e$ & 0.1823 & 0.5374 & 0.0086 & 0.067 \\
H(5A) & $4 e$ & 0.2569 & 0.7473 & -0.0854 & 0.094 \\
H(5B) & $4 e$ & 0.1579 & 0.5867 & -0.0888 & 0.094 \\
H(6A) & $4 e$ & 0.1285 & 0.8945 & -0.0203 & 0.083 \\
H(6B) & $4 e$ & 0.0295 & 0.7340 & -0.0237 & 0.083 \\
H(10) & $4 e$ & 0.5013 & 1.0418 & 0.1541 & 0.073 \\
H(13A) & $4 e$ & 0.1784 & 0.4839 & 0.1213 & 0.126 \\
H(13B) & $4 e$ & 0.0342 & 0.5001 & 0.1225 & 0.126 \\
H(13C) & $4 e$ & 0.1161 & 0.5934 & 0.1681 & 0.126 \\
H(15A) & $4 e$ & 0.0021 & 0.9687 & 0.1097 & 0.085 \\
H(15B) & $4 e$ & 0.0016 & 1.1382 & 0.1463 & 0.085 \\
\hline
\end{tabular}


Table 2. Continued.

\begin{tabular}{llllll}
\hline Atom & Site & $x$ & $y$ & $z$ & $U_{\text {iso }}$ \\
\hline H(16A) & 4e & -0.1901 & 1.0054 & 0.1519 & 0.091 \\
H(16B) & $4 e$ & -0.1280 & 0.8301 & 0.1713 & 0.091 \\
H(17) & $4 e$ & -0.0224 & 0.8169 & 0.2648 & 0.080 \\
H(18A) & $4 e$ & -0.0267 & 1.1320 & 0.3258 & 0.134 \\
\hline
\end{tabular}

Table 2. Continued.

\begin{tabular}{llrlll}
\hline Atom & Site & \multicolumn{1}{c}{$x$} & $y$ & $z$ & $U_{\text {iso }}$ \\
\hline $\mathrm{H}(18 \mathrm{~B})$ & $4 e$ & 0.0362 & 0.9604 & 0.3473 & 0.134 \\
$\mathrm{H}(18 \mathrm{C})$ & $4 e$ & -0.1076 & 0.9689 & 0.3395 & 0.134 \\
$\mathrm{H}(19 \mathrm{~A})$ & $4 e$ & 0.1046 & 1.1283 & 0.2405 & 0.078 \\
$\mathrm{H}(19 \mathrm{~B})$ & $4 e$ & 0.1688 & 0.9542 & 0.2595 & 0.078 \\
\hline
\end{tabular}

Table 3. Atomic coordinates and displacement parameters (in $\AA^{2}$ ).

\begin{tabular}{|c|c|c|c|c|c|c|c|c|c|c|}
\hline Atom & Site & $x$ & $y$ & 2 & $U_{11}$ & $U_{22}$ & $U_{33}$ & $U_{12}$ & $U_{13}$ & $U_{23}$ \\
\hline $\mathrm{Cl}(1)$ & $4 e$ & $0.88042(7)$ & $0.4242(1)$ & $0.22628(4)$ & $0.0750(5)$ & $0.0897(6)$ & $0.0920(6)$ & $-0.0022(4)$ & $0.0201(4)$ & $0.0065(5)$ \\
\hline$F(1)$ & $4 e$ & $0.3214(2)$ & $1.1250(3)$ & $0.21166(7)$ & $0.080(1)$ & $0.101(1)$ & $0.091(1)$ & $-0.016(1)$ & $0.0005(9)$ & $-0.041(1)$ \\
\hline$N(1)$ & $4 e$ & $0.3226(2)$ & $0.7200(3)$ & $0.02170(8)$ & $0.043(1)$ & $0.053(1)$ & $0.051(1)$ & $-0.0046(9)$ & $-0.0094(9)$ & $0.003(1)$ \\
\hline $\mathbf{N}(2)$ & $4 e$ & $0.1022(2)$ & $0.9448(3)$ & $0.18070(8)$ & $0.052(1)$ & $0.076(2)$ & $0.048(1)$ & $0.007(1)$ & $-0.009(1)$ & $0.000(1)$ \\
\hline $\mathbf{N}(3)$ & $4 e$ & $-0.1249(2)$ & $1.0148(3)$ & $0.23152(9)$ & $0.055(1)$ & $0.079(2)$ & $0.061(1)$ & $0.002(1)$ & $0.002(1)$ & $0.003(1)$ \\
\hline$O(1)$ & $4 e$ & $0.6433(2)$ & $0.9412(3)$ & $0.08002(8)$ & $0.047(1)$ & $0.086(2)$ & $0.089(1)$ & $-0.015(1)$ & $-0.012(1)$ & $-0.002(1)$ \\
\hline$O(2)$ & $4 e$ & $0.7528(2)$ & $0.8061(3)$ & $-0.0021(1)$ & $0.049(1)$ & $0.086(2)$ & $0.108(2)$ & $-0.007(1)$ & $0.003(1)$ & $0.005(1)$ \\
\hline$O(3)$ & $4 e$ & $0.6461(2)$ & $0.6565(3)$ & $-0.06480(9)$ & $0.065(1)$ & $0.085(2)$ & $0.074(1)$ & $0.005(1)$ & $0.007(1)$ & $0.005(1)$ \\
\hline$O(4)$ & $4 e$ & $0.1165(2)$ & $0.7133(3)$ & $0.09478(7)$ & $0.048(1)$ & $0.085(1)$ & $0.061(1)$ & $-0.0152(9)$ & $-0.0035(8)$ & $-0.002(1)$ \\
\hline$C(1)$ & $4 e$ & $0.6489(2)$ & $0.7378(4)$ & $-0.0223(1)$ & $0.049(2)$ & $0.061(2)$ & $0.074(2)$ & $0.002(1)$ & $-0.002(1)$ & $0.019(2)$ \\
\hline$C(2)$ & $4 e$ & $0.5393(2)$ & $0.7701(3)$ & $0.0131(1)$ & $0.046(1)$ & $0.051(2)$ & $0.062(2)$ & $-0.001(1)$ & $-0.006(1)$ & $0.013(1)$ \\
\hline$C(3)$ & $4 e$ & $0.4290(2)$ & $0.7024(3)$ & $-0.0050(1)$ & $0.051(2)$ & $0.053(2)$ & $0.054(1)$ & $0.001(1)$ & $-0.004(1)$ & $0.006(1)$ \\
\hline$C(4)$ & $4 e$ & $0.2114(2)$ & $0.6496(4)$ & $-0.0055(1)$ & $0.047(1)$ & $0.062(2)$ & $0.057(2)$ & $-0.009(1)$ & $-0.010(1)$ & $-0.002(1)$ \\
\hline$C(5)$ & $4 e$ & $0.1947(3)$ & $0.6794(5)$ & $-0.0666(1)$ & $0.058(2)$ & $0.118(3)$ & $0.059(2)$ & $-0.014(2)$ & $-0.014(1)$ & $-0.010(2)$ \\
\hline$C(6)$ & $4 e$ & $0.1149(2)$ & $0.7709(4)$ & $-0.0260(1)$ & $0.052(2)$ & $0.085(2)$ & $0.069(2)$ & $-0.004(1)$ & $-0.021(1)$ & $0.006(2)$ \\
\hline$C(7)$ & $4 e$ & $0.3216(2)$ & $0.8083(3)$ & $0.07298(9)$ & $0.045(1)$ & $0.046(1)$ & $0.050(1)$ & $-0.001(1)$ & $-0.010(1)$ & $0.006(1)$ \\
\hline$C(8)$ & $4 e$ & $0.4310(2)$ & $0.8911(3)$ & $0.0917(1)$ & $0.047(1)$ & $0.050(2)$ & $0.056(2)$ & $-0.003(1)$ & $-0.013(1)$ & $0.007(1)$ \\
\hline $\mathrm{C}(9)$ & $4 e$ & $0.5446(2)$ & $0.8707(3)$ & $0.0624(1)$ & $0.044(1)$ & $0.054(2)$ & $0.063(2)$ & $-0.004(1)$ & $-0.010(1)$ & $0.012(1)$ \\
\hline$C(10)$ & $4 e$ & $0.4290(2)$ & $0.9907(4)$ & $0.1404(1)$ & $0.050(2)$ & $0.065(2)$ & $0.067(2)$ & $-0.009(1)$ & $-0.011(1)$ & $-0.003(1)$ \\
\hline$C(11)$ & $4 e$ & $0.3229(3)$ & $1.0128(4)$ & $0.1675(1)$ & $0.063(2)$ & $0.061(2)$ & $0.059(2)$ & $-0.007(1)$ & $-0.011(1)$ & $-0.009(1)$ \\
\hline$C(12)$ & $4 e$ & $0.2173(2)$ & $0.8159(3)$ & $0.1065(1)$ & $0.042(1)$ & $0.056(2)$ & $0.052(1)$ & $-0.004(1)$ & $-0.009(1)$ & $0.002(1)$ \\
\hline$C(13)$ & $4 e$ & $0.1108(3)$ & $0.5598(4)$ & $0.1296(1)$ & $0.076(2)$ & $0.088(2)$ & $0.088(2)$ & $-0.032(2)$ & $0.013(2)$ & $0.003(2)$ \\
\hline$C(14)$ & $4 e$ & $0.2129(2)$ & $0.9275(3)$ & $0.1528(1)$ & $0.050(1)$ & $0.062(2)$ & $0.051(1)$ & $0.004(1)$ & $-0.006(1)$ & $0.005(1)$ \\
\hline$C(15)$ & $4 e$ & $-0.0022(2)$ & $1.0121(5)$ & $0.1476(1)$ & $0.060(2)$ & $0.099(2)$ & $0.053(2)$ & $0.015(2)$ & $-0.005(1)$ & $0.009(2)$ \\
\hline$C(16)$ & $4 e$ & $-0.1211(3)$ & $0.9559(5)$ & $0.1728(1)$ & $0.056(2)$ & $0.115(3)$ & $0.056(2)$ & $0.004(2)$ & $-0.010(1)$ & $0.000(2)$ \\
\hline$C(17)$ & $4 e$ & $-0.0188(3)$ & $0.9444(4)$ & $0.2653(1)$ & $0.067(2)$ & $0.082(2)$ & $0.050(2)$ & $0.000(2)$ & $-0.006(1)$ & $0.007(1)$ \\
\hline$C(18)$ & $4 e$ & $-0.0303(3)$ & $1.0072(5)$ & $0.3249(1)$ & $0.092(2)$ & $0.117(3)$ & $0.058(2)$ & $0.001(2)$ & $0.001(2)$ & $-0.001(2)$ \\
\hline$C(19)$ & $4 e$ & $0.0988(2)$ & $1.0024(4)$ & $0.2390(1)$ & $0.063(2)$ & $0.080(2)$ & $0.051(2)$ & $0.005(2)$ & $-0.010(1)$ & $0.000(1)$ \\
\hline
\end{tabular}

Acknowledgment. This project was supported by the Scientific Research Found of Hunan Provincial Education Department (grant no. 08c340).

\section{References}

1. Sheldrick, G. M.: SHELXS-97. Program for the Solution of Crystal Structures. University of Göttingen, Germany 1997.

2. Sheldrick, G. M.: SHELXL-97. Program for the Refinement of Crystal Structures. University of Göttingen, Germany 1997.
3. Sheldrick, G. M.: SHELXTL. Structure Determination Software Suite. Version 6.14. Bruker AXS, Madison, Wisconsin, USA 2000. 